\title{
Dibaryon with Highest Charm Number near Unitarity from Lattice QCD
}

\author{
Yan Lyu, ${ }^{1,2 \dagger}$ Hui Tong $\odot,{ }^{1,3, *}$ Takuya Sugiura, ${ }^{3}$ Sinya Aoki, ${ }^{4,2}$ Takumi Doi, ${ }^{2,3}$ \\ Tetsuo Hatsuda, ${ }^{3}$ Jie Meng $\odot,{ }^{1,5}$ and Takaya Miyamoto ${ }^{2}$ \\ ${ }^{1}$ State Key Laboratory of Nuclear Physics and Technology, School of Physics, Peking University, Beijing 100871, China \\ ${ }^{2}$ Quantum Hadron Physics Laboratory, RIKEN Nishina Center, Wako 351-0198, Japan \\ ${ }^{3}$ Interdisciplinary Theoretical and Mathematical Sciences Program (iTHEMS), RIKEN, Wako 351-0198, Japan \\ ${ }^{4}$ Center for Gravitational Physics, Yukawa Institute for Theoretical Physics, Kyoto University, Kyoto 606-8502, Japan \\ ${ }^{5}$ Yukawa Institute for Theoretical Physics, Kyoto University, Kyoto 606-8502, Japan
}

(Received 16 February 2021; revised 30 April 2021; accepted 2 July 2021; published 11 August 2021)

\begin{abstract}
A pair of triply charmed baryons, $\Omega_{c c c} \Omega_{c c c}$, is studied as an ideal dibaryon system by $(2+1)$-flavor lattice QCD with nearly physical light-quark masses and the relativistic heavy-quark action with the physical charm quark mass. The spatial baryon-baryon correlation is related to their scattering parameters on the basis of the HAL QCD method. The $\Omega_{c c c} \Omega_{c c c}$ in the ${ }^{1} S_{0}$ channel, taking into account the Coulomb repulsion with the charge form factor of $\Omega_{c c c}$, leads to the scattering length $a_{0}^{\mathrm{C}} \simeq-19 \mathrm{fm}$ and the effective range $r_{\text {eff }}^{\mathrm{C}} \simeq 0.45 \mathrm{fm}$. The ratio $r_{\text {eff }}^{\mathrm{C}} / a_{0}^{\mathrm{C}} \simeq-0.024$, whose magnitude is considerably smaller than that of the dineutron $(-0.149)$, indicates that $\Omega_{c c c} \Omega_{c c c}$ is located in the unitary regime.
\end{abstract}

DOI: 10.1103/PhysRevLett.127.072003

Introduction.-Quantum chromodynamics (QCD) is a fundamental theory of strong interaction and governs not only the interaction among quarks and gluons but also the interaction between color-neutral hadrons. In particular, the nucleon-nucleon $(N N)$ interaction, which shows a characteristic midrange attraction and a short-range repulsion, and the baryon-baryon $(B B)$ interactions are important for describing the nuclear structure and dense matter relevant to nuclear physics and astrophysics [1-5].

Although the deuteron is the only stable bound state composed of two nucleons, there are possible bound or resonant dibaryons with and without strange quarks [6-8]. Among others, $p \Omega$ (uudsss) [9] and $\Omega \Omega$ (ssssss) [10], which were predicted by lattice QCD (LQCD) simulations near the physical point [11], stimulate experimental searches in high energy hadron-hadron and heavy-ion collisions [8,12-14].

As originally pointed out by Bjorken [15], the triply charmed baryon (the charm number $C=3$ ) $\Omega_{c c c}$ is stable against the strong interaction and provides an ideal ground to study the perturbative and nonperturbative aspects of QCD in the baryonic sector. Although it has not been observed yet experimentally [16], there have been numerous LQCD studies on its mass and electromagnetic form factor (see [19] and references therein). Accordingly, it is timely to study the $\Omega_{c c c} \Omega_{c c c}$ as the simplest possible

Published by the American Physical Society under the terms of the Creative Commons Attribution 4.0 International license. Further distribution of this work must maintain attribution to the author(s) and the published article's title, journal citation, and DOI. Funded by SCOAP. system to study heavy-baryon interactions. The recent phenomenological study of $\Omega_{c c c} \Omega_{c c c}$ using the constituent quark model can be found in Ref. [20].

In this Letter, we present our study of a system with the charm number $C=6, \Omega_{c c c} \Omega_{c c c}$ in the ${ }^{1} S_{0}$ channel, for the first time from a first principle LQCD approach [21]. The reason we consider the $S$ wave and total spin $s=0$ system is that the Pauli exclusion between charm quarks at short distances does not operate in this channel, so that the maximum attraction is expected in comparison to other channels. It is of critical importance to examine the scattering parameters such as the scattering length and the effective range to unravel the properties of such heavy dibaryons near threshold. The HAL QCD method $[11,24,25]$, which treats the spatial correlation between two baryons on the lattice, provides a powerful tool for such analysis. Indeed, we show below that $\Omega_{c c c}^{++} \Omega_{c c c}^{++}\left({ }^{1} S_{0}\right)$ with strong interaction and Coulomb repulsion is located near unitarity $[26,27]$ just above the threshold with a large negative scattering length.

HAL QCD method.-The crucial steps in the HAL QCD method $[11,24,25]$ are to obtain the equal-time NambuBethe-Salpeter wave function $\psi(\boldsymbol{r})$, whose asymptotic behavior at a large distance reproduces the phase shifts, along with the corresponding two-baryon irreducible kernel $U\left(\boldsymbol{r}, \boldsymbol{r}^{\prime}\right)$. Since the same kernel $U\left(\boldsymbol{r}, \boldsymbol{r}^{\prime}\right)$ governs all the elastic scattering states, separating the ground state and the excited states on the lattice, which is exponentially difficult for baryon-baryon interactions [28,29], is not required to calculate the physical observables [25]. The normalized four-point function (the $R$ correlator) related to the NambuBethe-Salpeter wave function is defined as 


$$
\begin{aligned}
R(\boldsymbol{r}, t>0) & =\left\langle 0\left|\Omega_{c c c}(\boldsymbol{r}, t) \Omega_{c c c}(\mathbf{0}, t) \overline{\mathcal{J}}(0)\right| 0\right\rangle / e^{-2 m_{\Omega_{c c c}} t} \\
& =\sum_{n} A_{n} \psi_{n}(\boldsymbol{r}) e^{-\left(\Delta W_{n}\right) t}+O\left(e^{-\left(\Delta E^{*}\right) t}\right),
\end{aligned}
$$

where $\Delta W_{n}=2 \sqrt{m_{\Omega_{c c c}}^{2}+\boldsymbol{k}_{n}^{2}}-2 m_{\Omega_{c c c}}$ with the baryon mass $m_{\Omega_{c c c}}$ and the relative momentum $\boldsymbol{k}_{n} . O\left(e^{-\left(\Delta E^{*}\right) t}\right)$ denotes the contributions from the inelastic scattering states, with $\Delta E^{*}$ being the inelastic threshold, which are exponentially suppressed when $t \gg\left(\Delta E^{*}\right)^{-1} \sim \Lambda_{\mathrm{QCD}}^{-1}$ with $\Lambda_{\mathrm{QCD}} \sim 300 \mathrm{MeV} . \overline{\mathcal{J}}(0)$ is a source operator that creates two-baryon states with the charm number $C=6$ at Euclidean time $t=0$ and $A_{n}=\langle n|\overline{\mathcal{J}}(0)| 0\rangle$, with $|n\rangle$ representing the QCD eigenstates in a finite volume with $\Delta W_{n}<\Delta E^{*}$. In this study, we take a local interpolating operator, $\Omega_{c c c}(x) \equiv \epsilon^{l m n}\left[c_{l}^{T}(x) \mathcal{C} \gamma_{k} c_{m}(x)\right] c_{n}(x)$, where $l, m$, and $n$ stand for color indices, $\gamma_{k}$ is the Dirac matrix, and $\mathcal{C} \equiv \gamma_{4} \gamma_{2}$ is the charge conjugation matrix.

When contributions from the inelastic scattering states are negligible [ $t \gg\left(\Delta E^{*}\right)^{-1}$ ], the $R$ correlator satisfies [25]

$$
\left(\frac{1}{4 m_{\Omega_{c c c}}} \frac{\partial^{2}}{\partial t^{2}}-\frac{\partial}{\partial t}-H_{0}\right) R(\boldsymbol{r}, t)=\int d \boldsymbol{r}^{\prime} U\left(\boldsymbol{r}, \boldsymbol{r}^{\prime}\right) R\left(\boldsymbol{r}^{\prime}, t\right),
$$

where $H_{0}=-\nabla^{2} / m_{\Omega_{c c c}}$. By using the derivative expansion at low energies, $U\left(\boldsymbol{r}, \boldsymbol{r}^{\prime}\right)=V(r) \delta\left(\boldsymbol{r}-\boldsymbol{r}^{\prime}\right)+$ $\sum_{n=1} V_{2 n}(\boldsymbol{r}) \nabla^{2 n} \delta\left(\boldsymbol{r}-\boldsymbol{r}^{\prime}\right)$, the central potential $V(r)$ in the leading order is given as

$$
V(r)=R^{-1}(\boldsymbol{r}, t)\left(\frac{1}{4 m_{\Omega_{c c c}}} \frac{\partial^{2}}{\partial t^{2}}-\frac{\partial}{\partial t}-H_{0}\right) R(\boldsymbol{r}, t) .
$$

The spatial and temporal derivatives of $R(\boldsymbol{r}, t)$ on the lattice are calculated in central difference scheme by using the nearest neighbor points. To extract the total spin $s=0$, the following interpolating operator for the $\Omega_{c c c} \Omega_{c c c}$ system is adopted: $\left[\Omega_{c c c} \Omega_{c c c}\right]_{0}=$ $\frac{1}{2}\left(\Omega_{c c c}^{3 / 2} \Omega_{c c c}^{-3 / 2}-\Omega_{c c c}^{1 / 2} \Omega_{c c c}^{-1 / 2}+\Omega_{c c c}^{-1 / 2} \Omega_{c c c}^{1 / 2}-\Omega_{c c c}^{-3 / 2} \Omega_{c c c}^{3 / 2}\right)$. Here, the spin and its $z$ component of the interpolating operator $\Omega_{c c c}^{s_{z}}$ are $3 / 2$ and $s_{z}= \pm 3 / 2, \pm 1 / 2$, respectively, and $\Omega_{c c c}^{s_{z}}$ is constructed by spin projection as shown in Ref. [30]. To obtain the orbital angular momentum $L=0$ on the lattice, the projection to $A_{1}$ representation of the cubic group $S O(3, \mathbf{Z})$ is employed: $P^{A_{1}} R(\boldsymbol{r}, t)=$ $(1 / 24) \sum_{\mathcal{R}_{i} \in S O(3, \mathbf{Z})} R\left(\mathcal{R}_{i}[\boldsymbol{r}], t\right)$. Note that $V(r)$ in Eq. (3) contains a channel coupling effect such as ${ }^{1} S_{0^{-}}{ }^{5} D_{0}$ mixing and should be considered as an "effective" potential projected onto the $S$-wave state [31].

Lattice setup.- $(2+1)$-flavor gauge configurations are generated on the $L^{4}=96^{4}$ lattice with the Iwasaki gauge action at $\beta=1.82$ and nonperturbatively $O(a)$-improved Wilson quark action combined with stout smearing at nearly physical quark masses $\left(m_{\pi} \simeq\right.$ $146 \mathrm{MeV}$ and $m_{K} \simeq 525 \mathrm{MeV}$ ) [32]. The lattice cutoff is $a^{-1} \simeq 2.333 \mathrm{GeV} \quad(a \simeq 0.0846 \mathrm{fm}), \quad$ corresponding to $L a \simeq 8.1 \mathrm{fm}$, which is sufficiently large to accommodate two heavy baryons. For the charm quark, we employ the relativistic heavy quark (RHQ) action in order to remove the leading order and the next-to-leading order cutoff errors associated with the charm quark mass [33]. We use two sets (set 1 and set 2) of RHQ parameters determined in Ref. [34] so as to interpolate the physical charm quark mass and reproduce the dispersion relation for the spin-averaged $1 S$ charmonium, i.e., a weighted average of the spin-singlet state $\eta_{c}$ and the spin-triplet state $J / \Psi$.

For the source operator $\overline{\mathcal{J}}(0)$, we use the wall type with the Coulomb gauge fixing. We employ the periodic (Drichlet) boundary condition for spatial (temporal) direction. We use 112 gauge configurations, which are picked up 1 per 10 trajectories. In order to reduce statistical fluctuations, forward and backward propagations are averaged, and four time measurements are performed by shifting the source position along the temporal direction for each configuration. Then, the total measurements amount to 896 for each set. The statistical errors are estimated by the jackknife method with a bin size of 14 configurations. A comparison with a bin size of 7 configurations shows that the bin size dependence is small. The quark propagators are calculated by the Bridge ++ code [35], and the unified contraction algorithm is used to obtain the correlation functions [36].

Masses for spin-averaged $1 S$ charmonium $\left[\left(m_{\eta_{c}}+3 m_{J / \Psi}\right) / 4\right]$ and $\Omega_{c c c}$ baryon $\left(m_{\Omega_{c c c}}\right)$ calculated in set 1 and set 2 by using the single exponential fitting from the interval $t / a=25-35$ are listed in Table I, together with the values from linear interpolation $(0.3786 \times$ set $1+0.6214 \times$ set 2$)$ as well as the experimental value. Our result for $m_{\Omega_{c c c}}$ is consistent with 4789(6)(21) MeV obtained from (2+1)-flavor configurations generated by PACS-CS Collaboration [37]. We have checked that our results for hadron masses are unchanged within errors by the fitting interval $t / a=30-35$.

Numerical results.-The $\Omega_{c c c} \Omega_{c c c}$ potential $V(r)$ in the ${ }^{1} S_{0}$ channel from the interpolation between set 1 and set 2 is

TABLE I. Spin-averaged $1 S$ charmonium mass $\left[\left(m_{\eta_{c}}+3 m_{J / \Psi}\right) / 4\right]$ and the $\Omega_{c c c}$ mass $\left(m_{\Omega_{c c c}}\right)$ calculated in set 1 and set 2 with the statistical errors. The third row shows the interpolated values obtained from set 1 and set 2 . The experimental value of $\left(m_{\eta_{c}}+3 m_{J / \Psi}\right) / 4$ is shown in the last row.

\begin{tabular}{lcc}
\hline \hline & $\left(m_{\eta_{c}}+3 m_{J / \Psi}\right) / 4[\mathrm{MeV}]$ & $m_{\Omega_{c c c}}[\mathrm{MeV}]$ \\
\hline Set 1 & $3096.6(0.3)$ & $4837.3(0.7)$ \\
Set 2 & $3051.4(0.3)$ & $4770.2(0.7)$ \\
Interpolation & $3068.5(0.3)$ & $4795.6(0.7)$ \\
Experimental & $3068.5(0.1)$ & $\cdots$ \\
\hline \hline
\end{tabular}




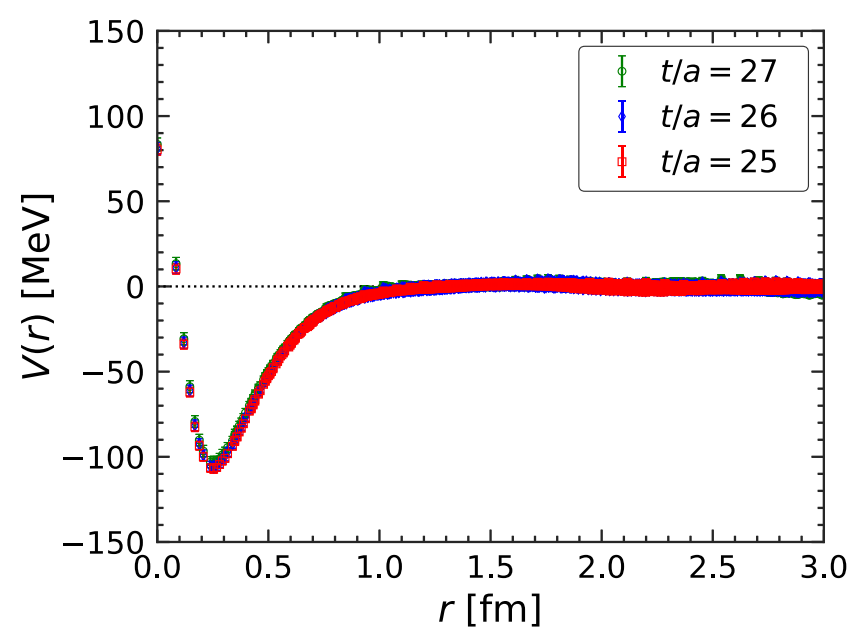

FIG. 1. The $\Omega_{c c c} \Omega_{c c c}$ potential $V(r)$ in the ${ }^{1} S_{0}$ channel as a function of separation $r$ at Euclidean time $t / a=25$ (red square), 26 (blue diamond), and 27 (green circle).

shown in Fig. 1 for $t / a=25,26$, and 27 [see the Supplemental Material [38] for the $t$ dependence of $V(r)$ in a wide range of $t]$. Since the potentials from set 1 and set 2 are found to be consistent within statistical errors, the uncertainty in the interpolation is negligible. Our choice $t / a=26$ corresponds to $t \simeq 2.2 \mathrm{fm}$; this is large enough in comparison to the typical length scale $\Lambda_{\mathrm{QCD}}^{-1} \sim 0.7 \mathrm{fm}$ characterizing the inelastic states and is small enough to avoid large statistical errors. We find that the potentials for $t / a=25,26$, and 27 are consistent with each other within statistical errors. This indicates that systematic errors due to inelastic states and higher order terms of the derivative expansion do not largely exceed the size of statistical errors [25], as we show below.

We find that the potential $V(r)$ is repulsive at short range and attractive at midrange, which has the same qualitative behaviors with the $N N$ potential [39] and the $\Omega \Omega$ potential [10]. The magnitude of the potential in the repulsive region $r<0.25 \mathrm{fm}$ [corresponding to $d V(r) / d r<0$ ] for $\Omega_{c c c} \Omega_{c c c}$ is an order of magnitude smaller than that of $\Omega \Omega$ obtained by the same method [10]. This may be qualitatively explained by the phenomenological quark model [40] as the color-magnetic interaction between constituent quarks is proportional to the square of the reciprocal constituent quark mass. Qualitatively, $V_{\mathrm{cm}}^{c c} / V_{\mathrm{cm}}^{s s}=\left(m_{s}^{*} / m_{c}^{*}\right)^{2} \sim(500 / 1500)^{2} \sim 0.1$, where $V_{\mathrm{cm}}^{f f^{\prime}}$ is the color-magnetic interaction between the quarks with flavor $f$ and $f^{\prime}$, with $m_{f}^{*}$ being the constituent quark mass. On the other hand, the attraction in the region $r>0.25 \mathrm{fm}$ [corresponding to $d V(r) / d r>0$ ] may originate from the exchange of charmed mesons or rather be attributed to the direct exchange of charm quarks and/or multiple gluons. As can be seen in Fig. 1, the range of the potential is much smaller than the size of the lattice volume, indicating that the finite volume artifact is negligible.
In order to convert the potential to physical observables such as the scattering phase shifts and binding energy, we perform the uncorrelated fit for $V(r)$ in Fig. 1 in the range $r \leq 2.5 \mathrm{fm}$ by threerange Gaussians, $V_{\text {fit }}(r)=\sum_{i=1,2,3} \alpha_{i} \exp \left(-\beta_{i} r^{2}\right)$. Fitting parameters with $t / a=26$, for example, are $\left(\alpha_{1}, \alpha_{2}, \alpha_{3}\right)=$ (239.5(3.0), -62.7(50.8),-98.8(50.3)) in $\mathrm{MeV}$ and $\left(\beta_{1}, \beta_{2}, \beta_{3}\right)=(48.5(1.4), 7.8(2.6), 3.4(0.8))$ in $\mathrm{fm}^{-2}$ with an accuracy of $\chi^{2} /$ d.o.f. $\sim 1.05$.

In Fig. 2, we show the $\Omega_{c c c} \Omega_{c c c}$ scattering phase shifts $\delta_{0}$ in the ${ }^{1} S_{0}$ channel calculated by solving the Schrödinger equation with the potential $V(r)$ at $t / a=25,26$, and 27. The relativistic kinetic energy is defined as $E_{\mathrm{CM}}=2 \sqrt{k^{2}+m_{\Omega_{c c c}}^{2}}-2 m_{\Omega_{c c c}}$, with a momentum $k$ in the center of mass frame. The error bands reflect the statistical uncertainty of $V(r)$. In all three cases, the phase shifts start from $180^{\circ}$ at $E_{\mathrm{CM}}=0$, which indicates the existence of a bound state in $\Omega_{c c c} \Omega_{c c c}$ system without Coulomb repulsion.

The low-energy scattering parameters are extracted by using the effective range expansion up to the next-toleading order, $k \cot \delta_{0}=-1 / a_{0}+\frac{1}{2} r_{\text {eff }} k^{2}+O\left(k^{4}\right)$, where $a_{0}$ and $r_{\text {eff }}$ are the scattering length and the effective range, respectively. The results are

$$
\begin{aligned}
a_{0} & =1.57(0.08)\left({ }_{-0.04}^{+0.12}\right) \mathrm{fm}, \\
r_{\text {eff }} & =0.57(0.02)\left({ }_{-0.00}^{+0.01}\right) \mathrm{fm} .
\end{aligned}
$$

The central values and the statistical errors in the first parentheses are obtained at $t / a=26$, while the systematic errors in the last parentheses are estimated from the values at $t / a=25,26$, and 27, which originates from the inelastic states and the higher order terms of the derivative expansion.

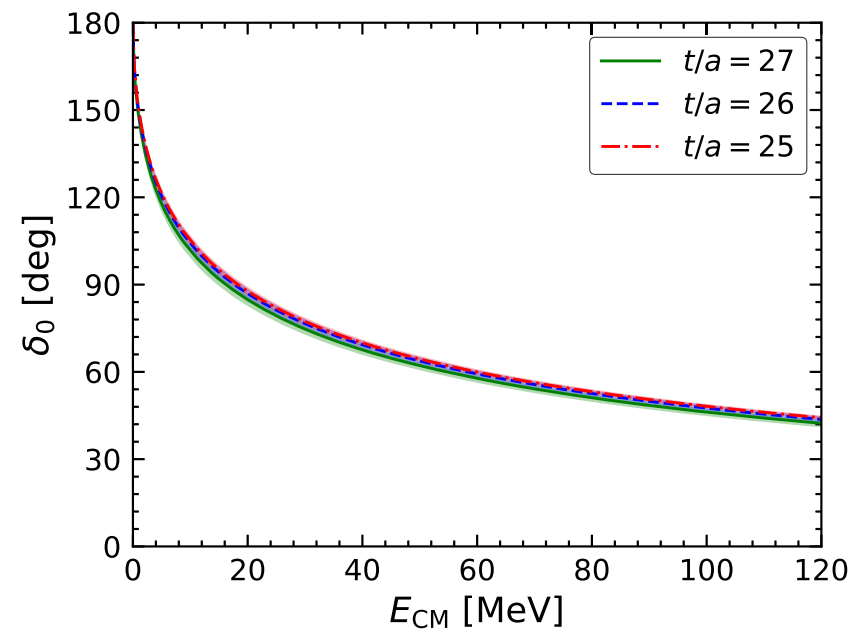

FIG. 2. The $\Omega_{c c c} \Omega_{c c c}$ scattering phase shifts $\delta_{0}$ in the ${ }^{1} S_{0}$ channel obtained from the potential $V(r)$ at $t / a=25,26$, and 27 as a function of the center of mass kinetic energy $E_{\mathrm{CM}}$. 
The binding energy $B$ and the root-mean-square distance $\sqrt{\left\langle r^{2}\right\rangle}$ of the bound $\Omega_{c c c} \Omega_{c c c}$ state are obtained from the potential $V(r)$ as

$$
\begin{aligned}
B & =5.68(0.77)\left({ }_{-1.02}^{+0.46}\right) \mathrm{MeV}, \\
\sqrt{\left\langle r^{2}\right\rangle} & =1.13(0.06)\left({ }_{-0.03}^{+0.80}\right) \mathrm{fm} .
\end{aligned}
$$

These results are consistent with the general formula for loosely bound states $[26,41]$ with scattering parameters $a_{0}$ and $r_{\text {eff }}: \quad B=\left(1-\sqrt{1-\left(2 r_{\text {eff }} / a_{0}\right)}\right)^{2} /\left(m_{\Omega_{c c c}} r_{\text {eff }}^{2}\right) \simeq$ $5.7 \mathrm{MeV}$ and $\sqrt{\left\langle r^{2}\right\rangle}=a_{0} / \sqrt{2} \simeq 1.1 \mathrm{fm}$.

Since the binding energy and the size of the bound state from the strong interaction are not large, we need to take into account the Coulomb repulsion $V^{\text {Coulomb }}(r)$ between $\Omega_{c c c}^{++}$s with finite spatial size. For this purpose, we consider the dipole form factor for $\Omega_{c c c}^{++}$according to the LQCD study on the charge distribution of heavy baryons [19]: In the coordinate space, it corresponds to an exponential charge distribution $\rho(r)=12 \sqrt{6} /\left(\pi r_{d}^{3}\right) e^{-2 \sqrt{6} r / r_{d}}$, where the charge radius $r_{d}=\sqrt{\left|\left\langle r^{2}\right\rangle_{\text {charge }}\right|}$ of $\Omega_{c c c}^{++}$is taken to be $r_{d}=0.410(6) \mathrm{fm}$ [19]. Then, we have

$V^{\text {Coulomb }}(r)=\alpha_{e} \iint d^{3} r_{1} d^{3} r_{2} \frac{\rho\left(r_{1}\right) \rho\left(\left|\vec{r}_{2}-\vec{r}\right|\right)}{\left|\vec{r}_{1}-\vec{r}_{2}\right|}=\frac{4 \alpha_{e}}{r} F(x)$,

where $x=2 \sqrt{6} r / r_{d}$ and $F(x)=1-e^{-x}[1+(11 / 16) x+$ $\left.(3 / 16) x^{2}+(1 / 48) x^{3}\right]$. The effective range expansion with Coulomb repulsion is written as

$k\left[C_{\eta}^{2} \cot \delta_{0}^{\mathrm{C}}(k)+2 \eta h(\eta)\right]=-\frac{1}{a_{0}^{\mathrm{C}}}+\frac{1}{2} r_{\mathrm{eff}}^{\mathrm{C}} k^{2}+O\left(k^{4}\right)$,

where $\delta_{0}^{\mathrm{C}}(k)$ is the phase shift in the presence of Coulomb repulsion, $\quad C_{\eta}^{2}=\left[2 \pi \eta /\left(e^{2 \pi \eta}-1\right)\right], \quad \eta=2 \alpha_{e} m_{\Omega_{c c c}} / k$, $h(\eta)=\operatorname{Re}[\Psi(i \eta)]-\ln (\eta)$, and $\Psi$ is the digamma function 42]]. To see the effect of the Coulomb repulsion, we vary $\alpha_{e}$ from zero to the physical value $\alpha_{e}^{\text {phys. }}=1 / 137.036$ below. Note that the systematic errors originated from the uncertainty in $r_{d}$ are found to be much smaller than the statistical errors and are neglected.

In Fig. 3, we show the inverse of scattering length $1 / a_{0}^{\mathrm{C}}$

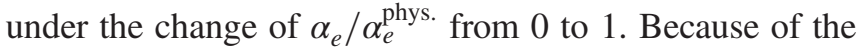
large cancellation between the attractive strong interaction and the Coulomb repulsion, the result at $\alpha_{e} / \alpha_{e}^{\text {phys. }}=1$ is located very close to unitarity with a large scattering length:

$$
\begin{aligned}
& a_{0}^{\mathrm{C}}=-19(7)\left(\begin{array}{c}
+7 \\
-6
\end{array}\right) \mathrm{fm}, \\
& r_{\text {eff }}^{\mathrm{C}}=0.45(0.01)\left({ }_{-0.00}^{+0.01}\right) \mathrm{fm} .
\end{aligned}
$$

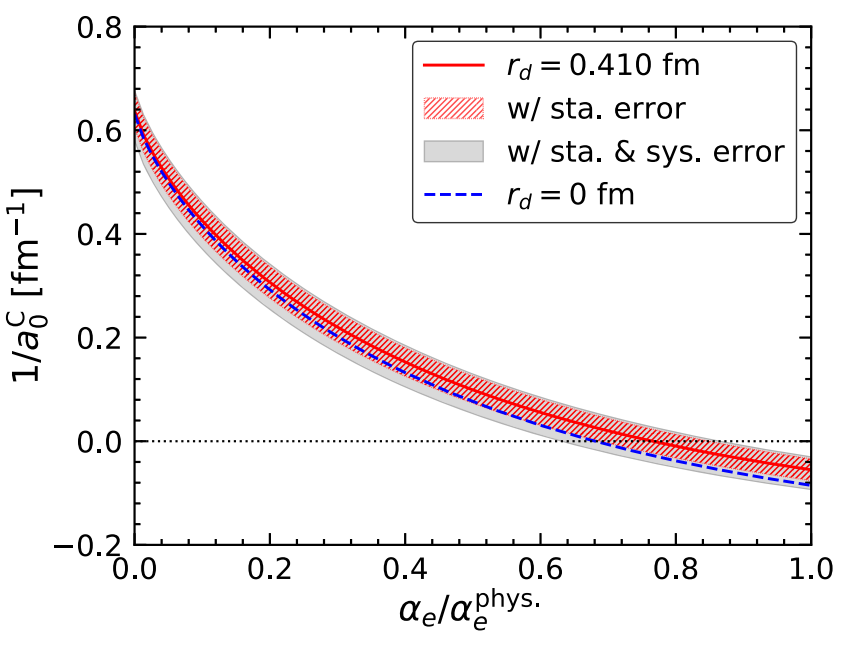

FIG. 3. The inverse of the scattering length $1 / a_{0}^{\mathrm{C}}$ as a function of $\alpha_{e} / \alpha_{e}^{\text {phys. }}$. The red solid line is the central values for $r_{d}=0.410 \mathrm{fm}$. The statistical errors are shown by the inner band (red), while the outer band (gray) corresponds to the statistical and systematic errors added in quadrature. The blue dashed line corresponds to the central values for $r_{d}=0 \mathrm{fm}$.

The ratio $r_{\text {eff }}^{\mathrm{C}} / a_{0}^{\mathrm{C}}=-0.024(0.010)\left({ }_{-0.014}^{+0.006}\right)$ is considerably smaller in magnitude than that of the dineutron $(-0.149)$.

In Fig. 4, we plot the dimensionless ratio $r_{\text {eff }} / a_{0}$ as a function of $r_{\text {eff }}$ for $\Omega_{c c c}^{++} \Omega_{c c c}^{++}\left({ }^{1} S_{0}\right)$ and $\Omega^{-} \Omega^{-}\left({ }^{1} S_{0}\right)$ with (without) Coulomb repulsion together with the experimental values for $N N\left({ }^{3} S_{1}-{ }^{3} D_{1}\right)$ [43] and $N N\left({ }^{1} S_{0}\right)[44,45]$. Note that we consider the Coulomb repulsion in $\Omega^{-} \Omega^{-}\left({ }^{1} S_{0}\right)$ with the charge radius $r_{d}=0.57 \mathrm{fm}$ for $\Omega^{-}[19,46]$. Among all those dibaryon systems, $\Omega_{c c c}^{++} \Omega_{c c c}^{++}\left({ }^{1} S_{0}\right)$ is the closest to unitarity. Note also that the nearly unitary binding of both $\Omega_{s s s}^{-} \Omega_{s s s}^{-}\left({ }^{1} S_{0}\right)$ and $\Omega_{c c c}^{++} \Omega_{c c c}^{++}\left({ }^{1} S_{0}\right)$ originates from a subtle cancellation among the potential energy, the kinetic energy, and the Coulomb repulsion.

Finally, we briefly discuss other possible systematic errors in this work: (i) The finite cutoff effect is $\mathcal{O}\left(\alpha_{s}^{2} a \Lambda_{\mathrm{QCD}},\left(a \Lambda_{\mathrm{QCD}}\right)^{2}\right)$ thanks to the RHQ action for the charm quark and the nonperturbative $O(a)$ improvement for light $(u, d, s)$ quarks, and thus amounts to $\mathcal{O}(1) \%$. (ii) In the vacuum polarization, light quark masses are slightly heavier than the physical ones and the charm quark loop is neglected. The former effect is expected to be small since light quarks are rather irrelevant for the $\Omega_{c c c} \Omega_{c c c}$ system. In fact, the range of the $\Omega_{c c c} \Omega_{c c c}$ potential is found to be shorter than $1 \mathrm{fm}$. The latter effect is suppressed due to the heavy charm quark mass and is typically $\mathcal{O}(1) \%$ [47]. These estimates for (i) and (ii) are also in line with the observation that our value of $m_{\Omega_{c c c}}$ is consistent with that in the literature or has a deviation of $\sim 1 \%$ at most, where we refer to LQCD studies by $(2+1)$-flavor at the physical point with finite $a$ [37], $(2+1)$-flavor with chiral and continuum extrapolation [48], and $(2+1+1)$-flavor with chiral and continuum extrapolation $[49,50]$. In the future, 


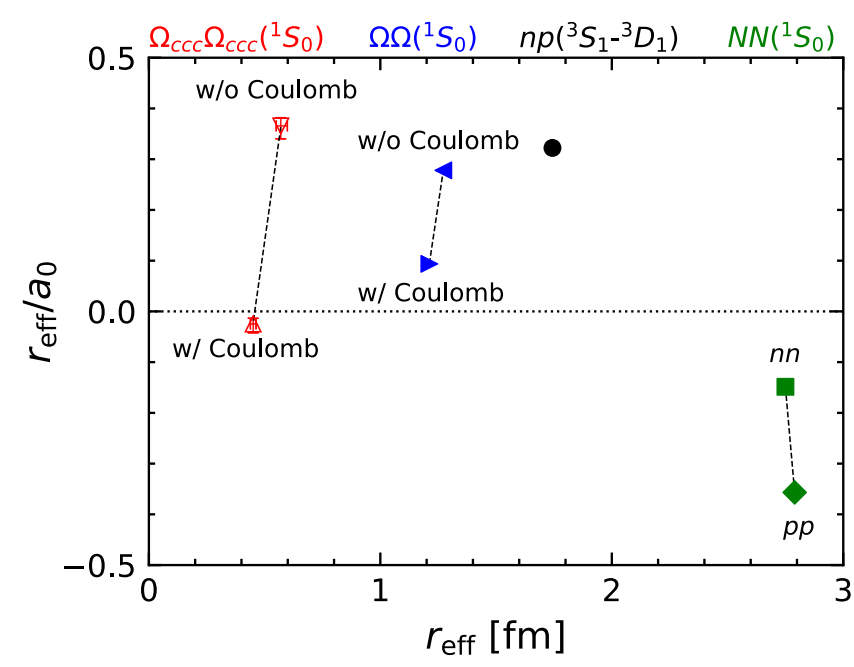

FIG. 4. The dimensionless ratio of the effective range $r_{\text {eff }}$ and the scattering length $a_{0}$ as a function of $r_{\text {eff }}$. The red up(down)pointing triangle and the blue right(left)-pointing triangle correspond to $\Omega_{c c c} \Omega_{c c c}$ system and $\Omega \Omega$ system in the ${ }^{1} S_{0}$ channel with (without) the Coulomb repulsion, respectively. The black circle represents $N N$ system in the ${ }^{3} S_{1}-{ }^{3} D_{1}$ channel. The green square $(n n)$ and diamond $(p p)$ correspond to $N N$ system in the ${ }^{1} S_{0}$ channel. The error bars for $\Omega_{c c c} \Omega_{c c c}$ are the quadrature of the statistical and systematic errors in Eqs. (4) and (8).

these systematic errors will be evaluated explicitly. Moreover, a finite volume analysis with proper projection of the sink operator [29] for the $\Omega_{c c c} \Omega_{c c c}$ system indicates that the truncation effect in the derivative expansion of $U\left(\boldsymbol{r}, \boldsymbol{r}^{\prime}\right)$ is small [38]. Further details will be reported elsewhere.

Summary and discussion.-In this Letter, we presented a first investigation on the scattering properties of the $\Omega_{c c c} \Omega_{c c c}$ on the basis of the $(2+1)$-flavor lattice QCD simulations with physical charm mass and nearly physical light quark masses. The potential for $\Omega_{c c c} \Omega_{c c c}\left({ }^{1} S_{0}\right)$ obtained by the time-dependent HAL QCD method without the Coulomb interaction shows a weak repulsion at short distances surrounded by a relatively strong attractive well, which leads to a most charming $(C=6)$ dibaryon with the binding energy $B \simeq 5.7 \mathrm{MeV}$ and the size $\sqrt{\left\langle r^{2}\right\rangle} \simeq 1.1 \mathrm{fm}$. By taking into account the Coulomb repulsion between $\Omega_{c c c}^{++}$s with their charge form factor obtained from LQCD, the $\Omega_{c c c}^{++} \Omega_{c c c}^{++}\left({ }^{1} S_{0}\right)$ system turns into the unitary region with $r_{\text {eff }}^{\mathrm{C}} / a_{0}^{\mathrm{C}} \simeq-0.024$. This provides good information toward the understanding of the interaction between heavy baryons. It will be interesting in future work to study $\Omega_{b b b}^{-} \Omega_{b b b}^{-}\left({ }^{1} S_{0}\right)$ w.r.t. revealing the quark mass dependence of the scattering parameters. Finally, our results may further stimulate future experimental activities to measure pairmomentum correlations of heavy baryons in high energy $p p, p A$, and $A A$ collisions $[8,14]$.
We thank the members of the HAL QCD Collaboration for technical support and stimulating discussions. We thank Yusuke Namekawa for providing the RHQ parameters. We thank members of PACS Collaboration for the gauge configuration generation conducted on the $\mathrm{K}$ computer at RIKEN. The lattice QCD measurements have been performed on HOKUSAI supercomputers at RIKEN. This work was partially supported by HPCI System Research Project (hp120281, hp130023, hp140209, hp150223, hp150262, hp160211, hp170230, hp170170, hp180117, hp190103). We thank ILDG/JLDG [51], which serves as an essential infrastructure in this study. We thank the authors of cuLGT code [52] for the gauge fixing. We thank Tatsumi Aoyama, Haozhao Liang, Shuangquan Zhang, and Pengwei Zhao for helpful discussions. Y. L., H. T., and J. M. were partially supported by the National Key R\&D Program of China (Contract Nos. 2017YFE0116700 and 2018YFA0404400) and the National Natural Science Foundation of China (NSFC) under Grant Nos. 11935003, 11975031, 11875075, and 12070131001. This work was partially supported by JSPS Grant Nos. JP18H05236, JP16H03978, JP19K03879, and JP18H05407 and the MOST-RIKEN Joint Project " $A b$ initio investigation in nuclear physics," "Priority Issue on Post-K computer" (Elucidation of the Fundamental Laws and Evolution of the Universe), "Program for Promoting Researches on the Supercomputer Fugaku" (Simulation for basic science: from fundamental laws of particles to creation of nuclei), and Joint Institute for Computational Fundamental Science (JICFuS).

Y. L. and H. T. contributed equally to this Letter and should be considered as co-first authors.

* Corresponding author. tongh16@pku.edu.cn †helvetia@pku.edu.cn

[1] E. Epelbaum, H.-W. Hammer, and U.-G. Meißner, Rev. Mod. Phys. 81, 1773 (2009).

[2] J. Meng, Relativistic Density Functional for Nuclear Structure (World Scientific, Singapore, 2016).

[3] S. Shen, H. Liang, W. H. Long, J. Meng, and P. Ring, Prog. Part. Nucl. Phys. 109, 103713 (2019).

[4] C. Drischler, W. Haxton, K. McElvain, E. Mereghetti, A. Nicholson, P. Vranas, and A. Walker-Loud, arXiv:1910 .07961 .

[5] H. Tong, P. Zhao, and J. Meng, Phys. Rev. C 101, 035802 (2020).

[6] H. Clement, Prog. Part. Nucl. Phys. 93, 195 (2017).

[7] A. Gal, Acta Phys. Pol. B 47, 471 (2016).

[8] S. Cho, T. Hyodo, D. Jido, C. M. Ko, S. H. Lee, S. Maeda, K. Miyahara, K. Morita, M. Nielsen, A. Ohnishi, T. Sekihara, T. Song, S. Yasui, and K. Yazaki, Prog. Part. Nucl. Phys. 95, 279 (2017). 
[9] T. Iritani, S. Aoki, T. Doi, F. Etminan, S. Gongyo, T. Hatsuda, Y. Ikeda, T. Inoue, N. Ishii, T. Miyamoto, and K. Sasaki, Phys. Lett. B 792, 284 (2019).

[10] S. Gongyo, K. Sasaki, S. Aoki, T. Doi, T. Hatsuda, Y. Ikeda, T. Inoue, T. Iritani, N. Ishii, T. Miyamoto, and H. Nemura (HAL QCD Collaboration), Phys. Rev. Lett. 120, 212001 (2018).

[11] S. Aoki and T. Doi, Front. Phys. 8, 307 (2020).

[12] K. Morita, S. Gongyo, T. Hatsuda, T. Hyodo, Y. Kamiya, and A. Ohnishi, Phys. Rev. C 101, 015201 (2020).

[13] S. Acharya et al. (ALICE Collaboration), Nature (London) 588, 232 (2020).

[14] L. Fabbietti, V. M. Sarti, and O. V. Doce, arXiv:2012.09806.

[15] J. Bjorken, AIP Conf. Proc. 132, 390 (1985).

[16] Recently, excited states of $C=1$ baryon $\Omega_{c}$ [17] and a $C=2$ baryon $\Xi_{c c}^{++}[18]$ were discovered at the CERN LHC.

[17] R. Aaij et al. (LHCb Collaboration), Phys. Rev. Lett. 118, 182001 (2017).

[18] R. Aaij et al. (LHCb Collaboration), Phys. Rev. Lett. 119, 112001 (2017).

[19] K. U. Can, G. Erkol, M. Oka, and T. T. Takahashi, Phys. Rev. D 92, 114515 (2015).

[20] H. Huang, J. Ping, X. Zhu, and F. Wang, arXiv:2011.00513.

[21] In the charm number $C=3$ sector, there exist a few recent studies on heavy dibaryons in LQCD [22] and in the constituent quark model [23].

[22] P. Junnarkar and N. Mathur, Phys. Rev. Lett. 123, 162003 (2019).

[23] J.-M. Richard, A. Valcarce, and J. Vijande, Phys. Rev. Lett. 124, 212001 (2020).

[24] N. Ishii, S. Aoki, and T. Hatsuda, Phys. Rev. Lett. 99, 022001 (2007).

[25] N. Ishii, S. Aoki, T. Doi, T. Hatsuda, Y. Ikeda, T. Inoue, K. Murano, H. Nemura, and K. Sasaki (HAL QCD Collaboration), Phys. Lett. B 712, 437 (2012).

[26] E. Braaten and H.-W. Hammer, Phys. Rep. 428, 259 (2006).

[27] C. Chin, R. Grimm, P. Julienne, and E. Tiesinga, Rev. Mod. Phys. 82, 1225 (2010).

[28] G. P. Lepage, in From Actions to Answers: Proceedings of the TASI 1989, edited by T. Degrand and D. Toussaint (World Scientific, Singapore, 1990).

[29] T. Iritani, S. Aoki, T. Doi, T. Hatsuda, Y. Ikeda, T. Inoue, N. Ishii, H. Nemura, and K. Sasaki (HAL QCD), J. High Energy Phys. 03 (2019) 007.

[30] M. Yamada, K. Sasaki, S. Aoki, T. Doi, T. Hatsuda, Y. Ikeda, T. Inoue, N. Ishii, K. Murano, and H. Nemura (HAL QCD Collaboration), Prog. Theor. Exp. Phys. 2015, 071B01 (2015).
[31] S. Aoki, T. Doi, T. Hatsuda, Y. Ikeda, T. Inoue, N. Ishii, K. Murano, H. Nemura, and K. Sasaki (HAL QCD Collaboration), Prog. Theor. Exp. Phys. 2012, $01 \mathrm{~A} 105$ (2012).

[32] K.-I. Ishikawa, N. Ishizuka, Y. Kuramashi, Y. Nakamura, Y. Namekawa, Y. Taniguchi, N. Ukita, T. Yamazaki, and T. Yoshie (PACS Collaboration), Proc. Sci., LATTICE2015 (2016) 075 [arXiv:1511.09222].

[33] S. Aoki, Y. Kuramashi, and S.-I. Tominaga, Prog. Theor. Phys. 109, 383 (2003).

[34] Y. Namekawa (PACS Collaboration), Proc. Sci., LATTICE2016 (2017) 125.

[35] http://bridge.kek.jp/Lattice-code/index_e.html.

[36] T. Doi and M. G. Endres, Comput. Phys. Commun. 184, 117 (2013).

[37] Y. Namekawa, S. Aoki, K.-I. Ishikawa, N. Ishizuka, K. Kanaya, Y. Kuramashi, M. Okawa, Y. Taniguchi, A. Ukawa, N. Ukita, and T. Yoshié (PACS-CS Collaboration), Phys. Rev. D 87, 094512 (2013).

[38] See Supplemental Material at http://link.aps.org/ supplemental/10.1103/PhysRevLett.127.072003 for the systematics related to the inelastic excited states and the derivative expansion of the nonlocal potential.

[39] T. Doi et al., Proc. Sci., LATTICE2016 (2017) 110 [arXiv:1702.01600].

[40] M. Oka, K. Shimizu, and K. Yazaki, Nucl. Phys. A464, 700 (1987).

[41] P. Naidon and S. Endo, Rep. Prog. Phys. 80, 056001 (2017).

[42] P. G. Burke, R-Matrix Theory of Atomic Collisions (Springer-Verlag, Berlin, 2011).

[43] R. W. Hackenburg, Phys. Rev. C 73, 044002 (2006).

[44] J. R. Bergervoet, P. C. van Campen, W. A. van der Sanden, and J. J. de Swart, Phys. Rev. C 38, 15 (1988).

[45] I. Slaus, Y. Akaishi, and H. Tanaka, Phys. Rep. 173, 257 (1989).

[46] In Ref. [10], $\Omega^{-}$was assumed to be pointlike in charge distribution, which overestimates the repulsion and increases the scattering length by $1 \mathrm{fm}$.

[47] S. Aoki et al. (Flavour Lattice Averaging Group), Eur. Phys. J. C 80, 113 (2020).

[48] Z. S. Brown, W. Detmold, S. Meinel, and K. Orginos, Phys. Rev. D 90, 094507 (2014).

[49] R. A. Briceño, H.-W. Lin, and D. R. Bolton, Phys. Rev. D 86, 094504 (2012).

[50] C. Alexandrou, V. Drach, K. Jansen, C. Kallidonis, and G. Koutsou, Phys. Rev. D 90, 074501 (2014).

[51] http://www.lqcd.org/ildg and http://www.jldg.org.

[52] M. Schrock and H. Vogt, Comput. Phys. Commun. 184, 1907 (2013). 